\title{
Dihydroartemisinin inhibits vascular endothelial growth factor-induced endothelial cell migration by a p38 mitogen-activated protein kinase-independent pathway
}

\author{
LING GUO $^{1,2}$, FENGYUN DONG ${ }^{1}$, YINGLONG HOU ${ }^{2}$, WEIDONG CAI $^{3}$, XIA ZHOU $^{4}$, \\ AI-LING HUANG ${ }^{5}$, MIN YANG $^{6}$, THADDEUS D. ALLEN ${ }^{7}$ and $\mathrm{JU} \mathrm{LIU}^{1}$ \\ ${ }^{1}$ Laboratory of Microvascular Medicine, Medical Research Center and Departments of ${ }^{2}$ Cardiology, ${ }^{3}$ Emergency Medicine, \\ ${ }^{4}$ Traditional Chinese Medicine and ${ }^{5}$ Nephrology, Shandong Provincial Qianfoshan Hospital, Shandong University, Jinan, \\ Shandong 250014; ${ }^{6}$ Department of Orthopedics, Xijing Hospital, The Fourth Military Medical University, \\ Xi'an, Shaanxi 710032, P.R. China; ${ }^{7}$ G.W. Hooper Research Foundation, \\ University of California, San Francisco, CA 94143-0552, USA
}

Received March 4, 2014; Accepted July 21, 2014

DOI: $10.3892 /$ etm.2014.1997

\begin{abstract}
Dihydroartemisinin (DHA), a semi-synthetic derivative of artemisinin, has been demonstrated to possess a strong antiangiogenic activity. However, the molecular mechanisms underlying this effect remain unclear. Endothelial cell (EC) migration is an essential component of angiogenesis, and the p38 mitogen-activated protein kinase (MAPK) signaling pathway plays a key role in the regulation of migration induced by vascular endothelial growth factor (VEGF). The aim of the present study was to investigate the effects of DHA on EC migration and the p38 MAPK signaling pathway. Human umbilical vein ECs (HUVECs) were treated with DHA and VEGF-induced migration was analyzed. The activation of p38 MAPK was detected by western blot analysis, and the migration assays were performed with a $\mathrm{p} 38$-specific inhibitor, SB203850. It was revealed that $20 \mu \mathrm{M}$ DHA significantly reduced $\mathrm{EC}$ migration in the transwell migration assay, wound healing assay and electrical cell-substrate impedance sensing real-time analysis. However, DHA did not affect p38 MAPK phosphorylation or expression. In the absence or presence of SB203850, DHA induced a similar proportional reduction of EC migration in the three migration assays. Therefore, the present study demonstrated that DHA inhibits VEGF-induced EC migration via a p38 MAPK-independent pathway.
\end{abstract}

Correspondence to: Professor Ju Liu, Laboratory of Microvascular Medicine, Medical Research Center, Shandong Provincial Qianfoshan Hospital, Shandong University, 16766 Jingshi Road, Jinan, Shandong 250014, P.R. China

E-mail: jliu0228@gmail.com

Key words: dihydroartemisinin, migration, p38 mitogen-activated protein kinase, endothelial cell, angiogenesis

\section{Introduction}

Artemisinin is a sesquiterpene lactone isolated from the Artemisia annua plant that has been used extensively as an antimalarial drug (1). Dihydroartemisinin (DHA) is the active metabolite of artemisinin compounds, and a semisynthetic derivative of artemisinin (2). DHA is an effective, water-soluble antimalarial drug, with fewer side-effects compared with other drugs (3). In addition, DHA exhibits strong antitumor and antiangiogenesis effects (4). However, the underlying mechanisms of DHA remain poorly understood.

Angiogenesis, the formation of new capillaries branching from existing vessels, plays a critical role in embryonic development, wound-healing and the menstrual cycle (5). Abnormal angiogenesis is associated with tumor growth, diabetes, rheumatoid arthritis and atherosclerosis (6). Under hypoxic conditions, tumor cells or other growing cells secrete growth factors, including vascular endothelial growth factor (VEGF) and basic fibroblast growth factor (bFGF). These growth factors activate endothelial cells (ECs) in nearby capillaries, leading to the migration of ECs branching out of the vessel (7). The cells proliferate and differentiate to form a network of new capillaries. It has been well established that solid tumor growth requires a neovascular network, which enables rapid proliferation of tumor cells by providing oxygen and nutrients (8).

EC migration is an essential component of angiogenesis (7). This motile process is regulated by chemotactic, haptotactic and mechanotactic stimuli (7). Typically, chemotaxis of ECs is driven by growth factors, including VEGF and bFGF, whereas haptotaxis is associated with increased EC motility in response to integrins binding to the extracellular matrix (5). The process requires the activation of several signaling pathways that converge on cytoskeletal remodeling (9).

Mitogen-activated protein kinase (MAPK) pathways constitute a large network of signaling cascades that regulate diverse physiological processes, including cell migration (10). The functions of MAPKs are mediated through 
the phosphorylation of substrates, including phospholipases, transcription factors and cytoskeletal proteins (11). To date, three major subfamilies of MAPKs have been well-characterized: Extracellular signal-regulated kinases (ERKs), p38 MAPKs and c-Jun N-terminal kinases (JNKs). The p38 pathway conveys the VEGF signal to microfilaments, inducing rearrangements in the actin cytoskeleton that regulate cell migration (12). Through the modulation of cell migration, p38 MAPK is an important regulator of angiogenesis (13).

In the present study, DHA was hypothesized to inhibit EC migration via the $\mathrm{p} 38$ MAPK pathway. Cell migration assays were performed on human umbilical vein ECs (HUVECs) with the addition of DHA, and the phosphorylation of the $\mathrm{p} 38$ MAPK was examined by western blot analysis. The role of $\mathrm{p} 38$ MAPK was further examined using an electrical cell-substrate impedance sensing (ECIS) system with the specific inhibitor, SB203850.

\section{Materials and methods}

Cell culture. HUVECs were purchased from the American Type Culture Collection (Manassas, VA, USA) and maintained in endothelial growth medium (EGM-2), supplemented with the EGM-2-MV bullet kit (Lonza, Basel, Switzerland) and antibiotics (100 IU/ml penicillin and $100 \mu \mathrm{g} / \mathrm{ml}$ streptomycin), in a humidified atmosphere at $37^{\circ} \mathrm{C}$ and $5 \% \mathrm{CO}_{2}$. DHA (sc-211332; Santa Cruz Biotechnology, Inc., Santa Cruz, CA, USA), anisomycin (sc-3524, Santa Cruz Biotechnology, Inc.) and SB203850 (Cell Signaling Technology, Inc., Beverly, MA, USA) were dissolved in dimethyl sulfoxide (DMSO).

Boyden chamber migration assay. Cell migration assays were performed using modified 24-well Boyden chambers (Costar, Acton, MA, USA), containing a polycarbonate membrane with $8.0-\mu \mathrm{m}$ pores. HUVECs were starved in basic EGM-2 (serum/growth factor free) overnight at $37^{\circ} \mathrm{C}$, prior to being harvested with trypsin and resuspended in basic EGM-2. The single cell suspensions with $20 \mu \mathrm{M}$ DHA or $20 \mu \mathrm{M}$ SB203850 were seeded at $1 \times 10^{5}$ cells/well in the upper chamber, while $0.5 \mathrm{ml}$ EGM-2 with $20 \mathrm{ng} / \mathrm{ml}$ VEGF was added to the bottom chamber as chemoattractants. After $24 \mathrm{~h}$ incubation, the migrated cells on the bottom surface were stained with $0.1 \%$ crystal violet (Santa Cruz Biotechnology, Inc.) and counted under an Olympus LCX100 Imaging system (Olympus Corporation, Tokyo, Japan).

Wound healing migration assay. HUVECs were grown to confluence in 24-well plates and starved for $2 \mathrm{~h}$. The media were changed to basic endothelial growth basal medium (EBM-2), supplemented with $100 \mathrm{ng} / \mathrm{ml}$ VEGF, and a scratch (wound) was made across the monolayer using a sterile pipette tip. DHA was added to the culture medium with a final concentration of $20 \mu \mathrm{M}$. Images of the wells were captured at fixed points to record the area of clearing at time 0 and $8 \mathrm{~h}$, and ImageJ software (NIH, Bethesda, MD, USA) was used to quantitate the cleared area.

Western blot analysis. HUVECs treated with $20 \mu \mathrm{M}$ DHA were collected at different time points. To generate a positive control for p38 MAPK activation, a group of HUVECs were treated with $1 \mu \mathrm{g} / \mathrm{ml}$ anisomycin for $1 \mathrm{~h}$. Cell lysates were prepared in radioimmunoprecipitation assay buffer [20 mM Tris (pH 7.5), 150 mM NaCl, 50 mM NaF, 1\% NP-40, $0.1 \%$ deoxycholate, $0.1 \%$ SDS and $1 \mathrm{mM}$ EDTA] (Santa Cruz Biotechnology, Inc.), supplemented with $1 \mathrm{mM}$ phenylmethylsulfonyl fluoride and $1 \mu \mathrm{g} / \mathrm{ml}$ leupeptin (Santa Cruz Biotechnology, Inc.). Cleared cell lysates were subjected to SDS-PAGE using 10\% polyacrylamide gel and transferred to polyvinylidene fluoride membranes. Membranes were blocked with $2.5 \%$ non-fat milk, and incubated with primary antibodies at $4^{\circ} \mathrm{C}$ overnight in phosphate-buffered saline Tween-20 (Santa Cruz Biotechnology, Inc.). The primary antibodies used were total-p38, phospho-p38 (Cell Signaling Technology, Inc.) and $\beta$-actin (Sigma-Aldrich, St. Louis, MO, USA). Immunoreactivity was visualized with horseradish peroxidase-conjugated secondary antibodies and an enhanced chemiluminescence reagent (Santa Cruz Biotechnology, Inc.). The blots were analyzed using a Bio-Rad imaging system (Bio-Rad, Hercules, CA, USA).

ECIS migration analysis. Real-time EC migration was measured using the ECIS technique (ECIS model 1600; Applied BioPhysics, Troy, NY, USA). Briefly, eight-well ECIS arrays $(8 \mathrm{~W} 10 \mathrm{E}+)$ were coated with fibronectin (Invitrogen Life Technologies, Carlsbad, CA, USA). HUVECs were plated at a confluent density to form monolayers directly on top of the electrodes. Next, an elevated voltage pulse of $40 \mathrm{kHz}$ was applied to the arrays for $30 \mathrm{sec}$, which resulted in the death and detachment of cells from the electrodes. As a result, the wound was healed by the surrounding cells. Following treatment with DHA or SB203850, an alternating current was applied to the cells across the electrodes and the electrical resistance was recorded. Data plots are representative of triplicate experiments, with each graph showing the resistance readings from a separate well, at 40 distinct electrodes per well.

Statistical analysis. Statistically significant differences were assessed using a paired-samples t-test. All statistical analyses were performed using SPSS 19.0 statistical software (Chicago, IL, USA) with a significance level of $\mathrm{P}<0.05$.

\section{Results}

DHA inhibits EC migration. Boyden chamber-type cell migration assays were used to assess the effect of DHA on EC migration. A low concentration of $20 \mu \mathrm{M}$ DHA was used, since it had been demonstrated to be sufficient to inhibit angiogenesis in vitro (14). The number of HUVECs migrating across the polycarbonate membrane was significantly reduced in the groups treated with $20 \mu \mathrm{M}$ DHA $(33.76 \%, \mathrm{P}<0.01$; Fig. 1A and B). Cell migration during in vitro wound healing mimics the process of EC motility in vivo $(12,15)$. Thus, wound healing migration assays were also performed, and the migrated area of HUVECs was significantly reduced in the DHA-treated groups when compared with the groups treated with vehicle DMSO alone (40.97\%, P<0.01; Fig. 1C and D). Thus, these in vitro assays indicated that DHA induced the reduction of EC migration.

Activation of 38 MAPK is unaffected by DHA in ECs. In ECs, p38 MAPK activation by VEGF mediates actin reorganiza- 
A

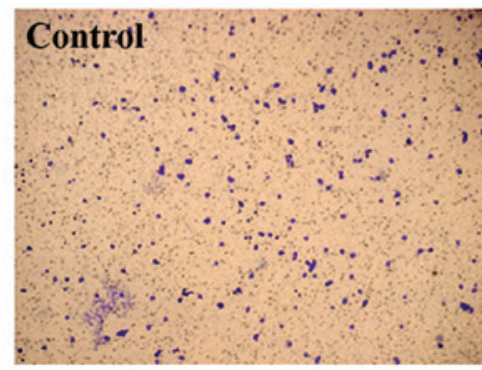

C

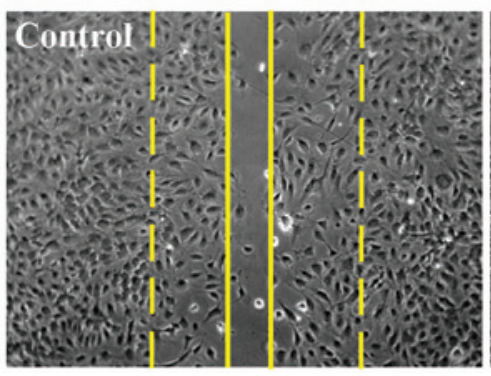

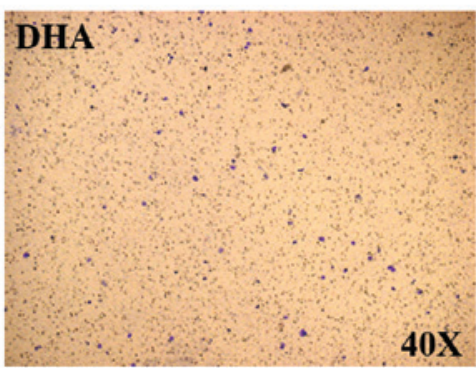

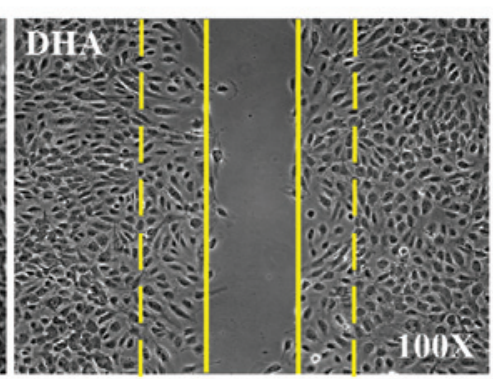

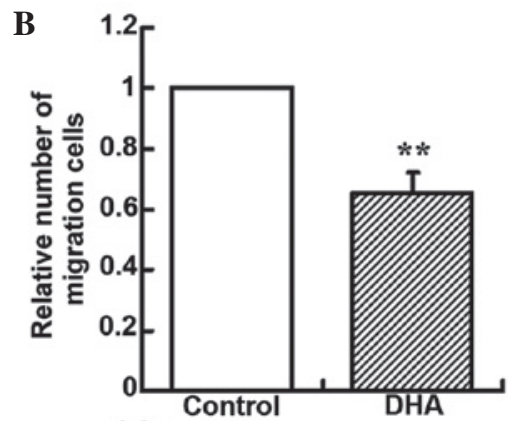

D

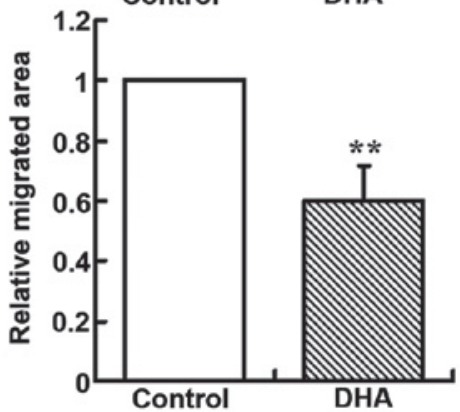

Figure 1. Cell migration of human umbilical vein endothelial cells treated with DHA. (A) Representative cell staining images following the transwell migration assay. (B) Number of cells migrating through the transwell membrane $(\mathrm{n}=6) ;{ }^{* *} \mathrm{P}<0.01$, vs. control. (C) Representative images of the wound healing assay. Dashed line indicates $0 \mathrm{~h}$ and the solid line indicates $8 \mathrm{~h}$. (D) Migrated area following the wound healing assay $(\mathrm{n}=12) ;{ }^{* * *} \mathrm{P}<0.01$, vs. control. DHA, dihydroartemisinin.

A

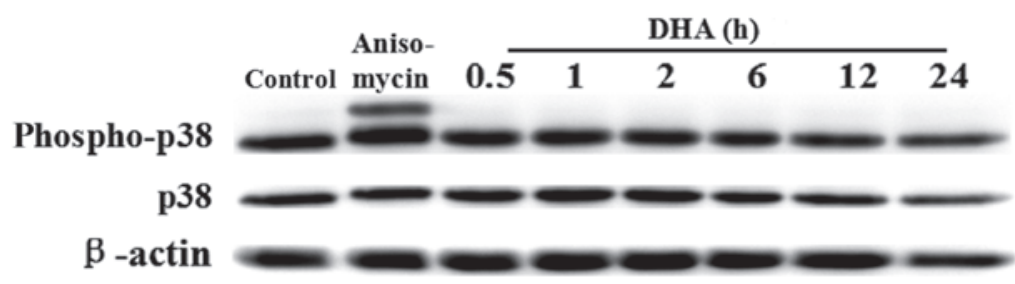

B

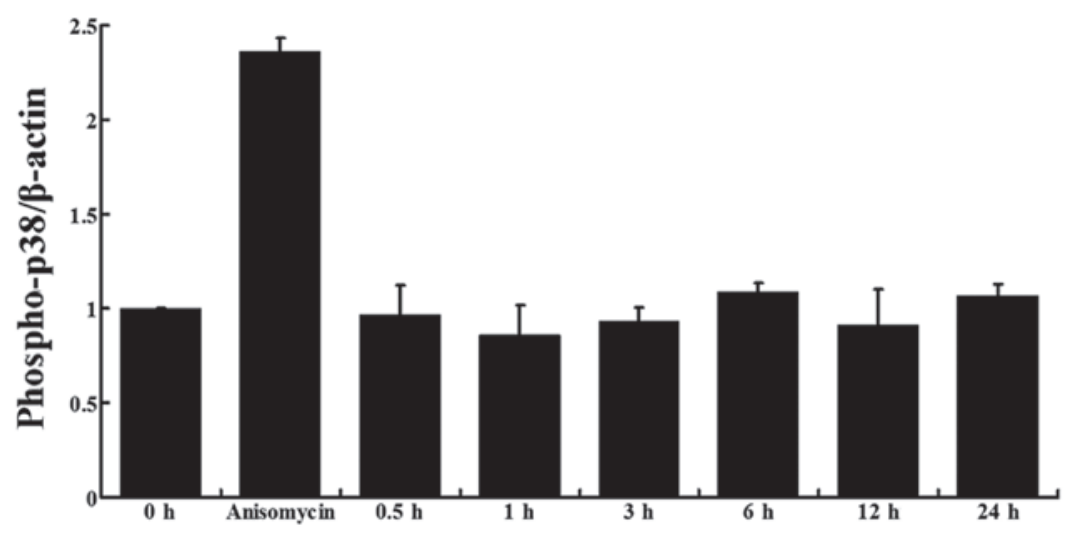

C

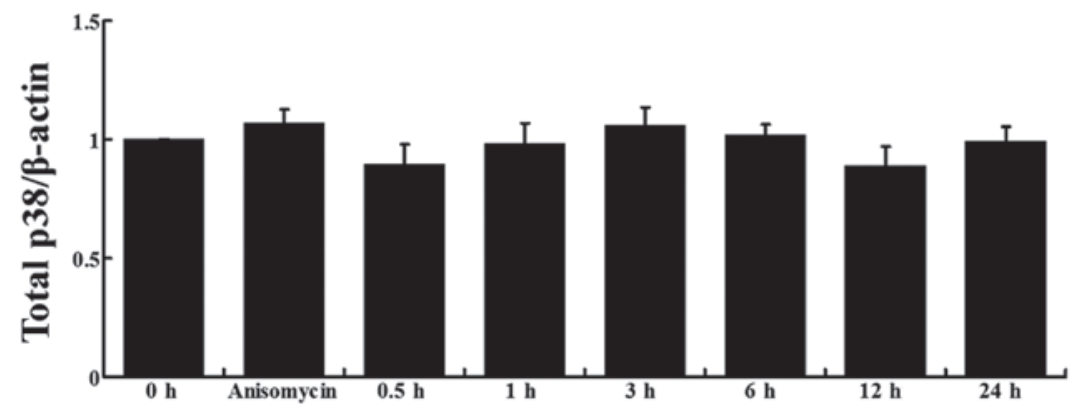

Figure 2. Effect of DHA on the activation of p38 mitogen-activated protein kinase (MAPK) in human umbilical vein endothelial cells (HUVECs). (A) Immunoblots of phospho-p38 MAPK, p38 MAPK and $\beta$-actin from protein samples of HUVECs treated with DHA at various time points. Densitometric analysis of the blots of (B) phospho-p38 MAPK and (C) total p38 MAPK. DHA, dihydroartemisinin. 
A

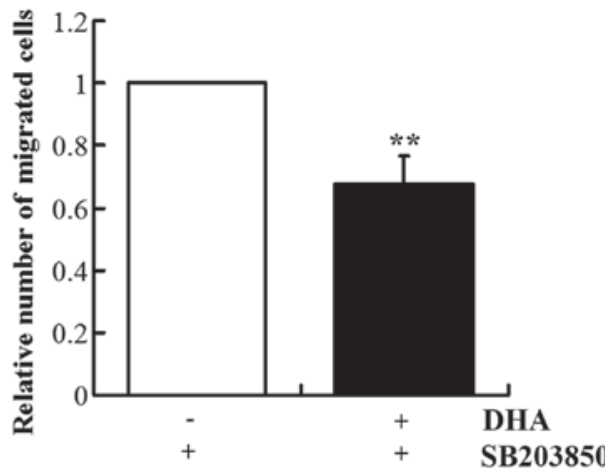

C

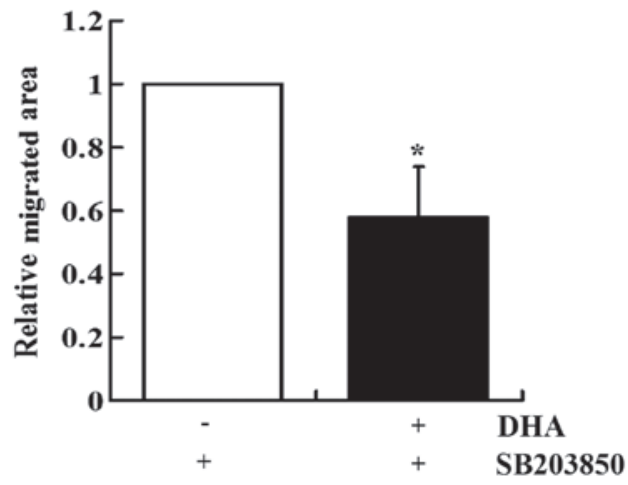

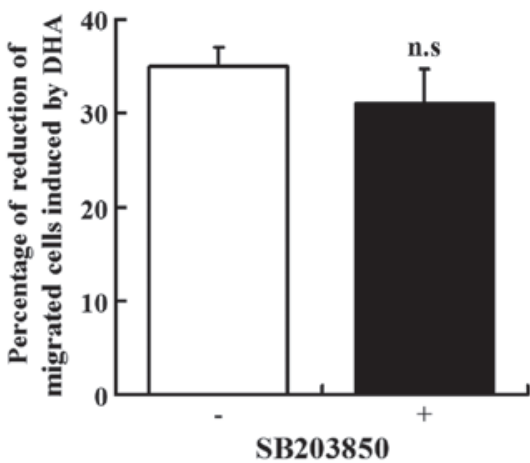

D

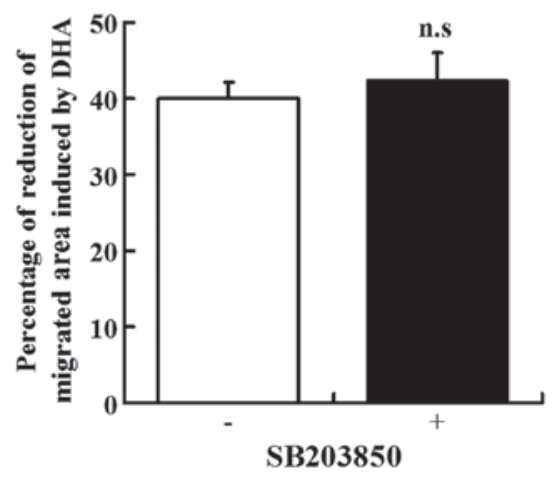

Figure 3. Effect of SB203850 on DHA-induced inhibition of endothelial cell migration. (A) Transwell migration assay of human umbilical vein endothelial cells (HUVECs) treated with SB203850 and DHA $(n=6) ;{ }^{* *} \mathrm{P}<0.01$. (B) Percentage reduction of migrated cells induced by DHA in the absence or presence of SB203850. (C) Wound healing assay of HUVECs treated with SB203850 and DHA ( $n=12)$; "P<0.05. (D) Percentage reduction in the migrated area induced by DHA in the absence or presence of SB203850. n.s., non-significant. DHA, dihydroartemisinin.

tion and cell migration (12). To examine the effects of DHA on p38 MAPK activation, HUVECs cultured in EBM-2, containing $100 \mathrm{ng} / \mathrm{ml}$ VEGF, were treated with $20 \mu \mathrm{M} \mathrm{DHA}$ and the protein expression was analyzed at different time points. HUVECs treated with anisomycin, a known activator of p38 MAPK, were used as a positive control (16). Western blot analysis showed that the total p38 and phospho-p38 MAPK protein expression levels remained unchanged during DHA treatment at all the indicated time points (Fig. 2A). Densitometric analysis further confirmed that DHA did not affect p38 MAPK activation or expression (Fig. 2B and C).

DHA-induced repression of EC migration is unaffected by SB203850. SB203850 is a pyridinyl imidazole derivative that inhibits p38 MAPK phosphorylation, but does not reduce p38 MAPK expression (17). The role of p38 MAPK in mediating the DHA-induced inhibition of EC migration was ascertained by examining the effect of SB203580 on the DHA-treated HUVECs. In the presence of SB203580, DHA also induced the reduction of EC migration in the Boyden chamber assay $(30.38 \%, \mathrm{P}<0.01)$ and wound healing assay $(43.04 \%, \mathrm{P}<0.05$; Fig. 3A and C). The levels of reduction induced by DHA in the two assays were similar in the absence or presence of SB203850 (Fig. 3B and D).

To capture the subtle alterations of cell migration, an ECIS system was implemented, which allowed for real-time measurements of the resistance caused by the migration of ECs. The transendothelial resistance was significantly reduced when HUVECs were treated with $20 \mu \mathrm{M}$ DHA $(\mathrm{P}<0.05$; Fig. 4A and B). Treatment with $20 \mu \mathrm{M}$ SB203580 also significantly decreased the transendothelial resistance $(\mathrm{P}<0.05)$; however, treatment with DHA and SB203850 induced an additional reduction in transendothelial resistance at 4,8 and $12 \mathrm{~h}$ (Fig. 4A and B). In the absence or presence of SB203850, the reduction of transendothelial resistance induced by DHA showed no statistically significant difference at any of the time points (Fig. 4C). Therefore, the inhibitory effects of DHA on EC migration are not mediated by the p38 MAPK pathway.

\section{Discussion}

Antiangiogenic therapy targeting ECs to block neovascularization has become an effective anticancer strategy (18). DHA, a widely used antimalarial drug with minimal side-effects, has been reported to be highly effective against tumor angiogenesis (14). The aim of the present study was to investigate the mechanisms underlying the effect of DHA on EC migration, a key process in angiogenesis. DHA was shown to inhibit EC migration in three types of migration assays. In addition, the role of the p38 MAPK signaling pathway in the effect of DHA was further investigated, and p38 MAPK activation was shown to not be affected by DHA. Furthermore, the p38 MAPK inhibitor, SB203850, did not inhibit or augment the DHA-induced reduction of EC migration. To the best of our knowledge, the present study is the first to investigate the signaling transduction pathways mediating the effects of the artemisinin family of drugs on EC migration.

EC migration is a dynamic and multistep process that plays a crucial role in the initiation and progression of 
A

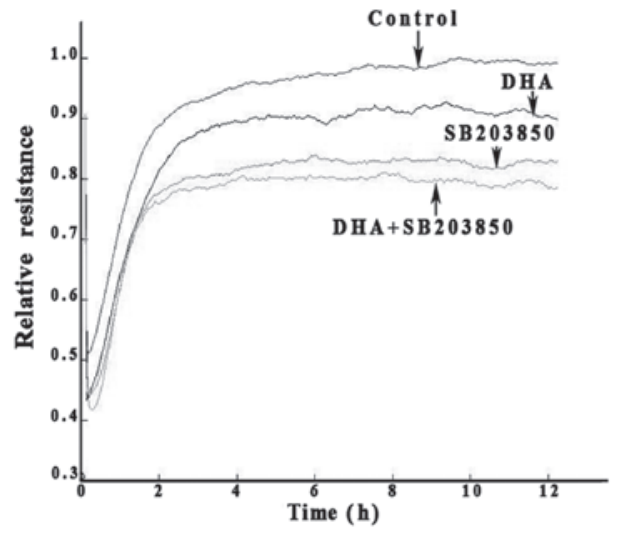

B

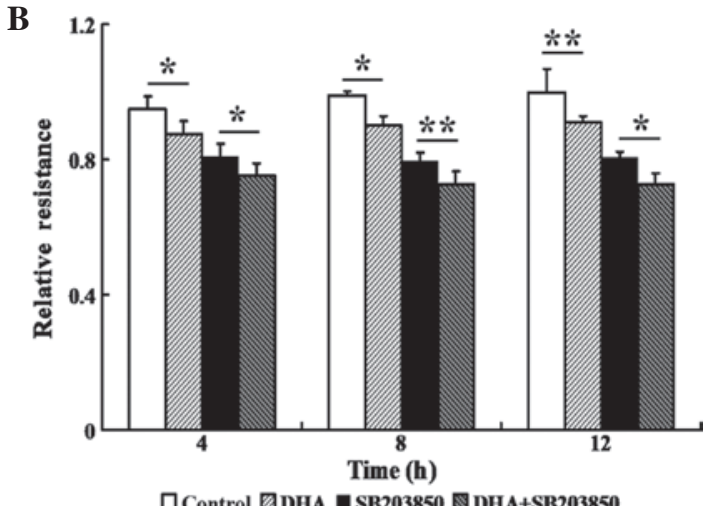

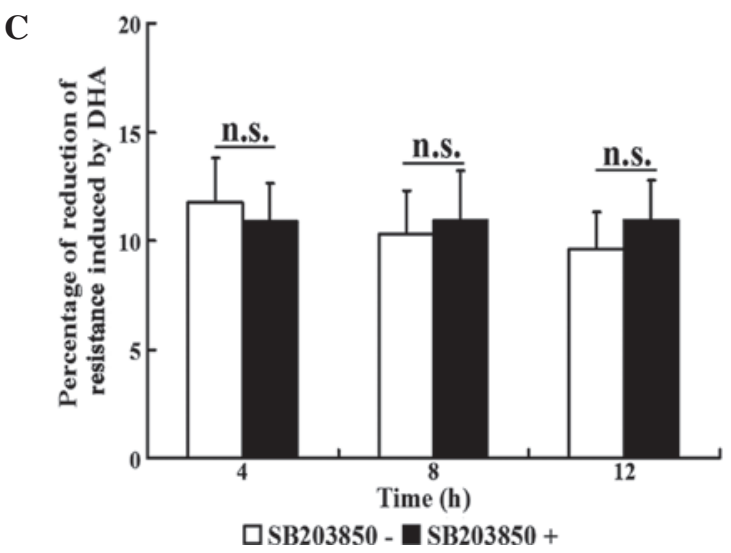

Figure 4. Transendothelial resistance determined using an electrical cell-substrate impedance sensing wound healing assay in human umbilical vein endothelial cells (HUVECs) treated with DHA and SB203850. (A) Real-time resistance measurement of the HUVEC monolayer treated with DHA and/or SB203850. (B) Bar graph showing the mean percentage of transendothelial resistance $(n=4) ;{ }^{*} \mathrm{P}<0.05 ;{ }^{* *} \mathrm{P}<0.01$. (C) Percentage reduction of resistance induced by DHA in the absence or presence of SB203850 (n=4). n.s., non-significant. DHA, dihydroartemisinin.

angiogenesis (19). Previous studies have demonstrated the antiangiogenetic effects of DHA and other artemisinin derivatives $(20,21)$. DHA has been shown to inhibit HUVEC migration in a dose-dependent manner (14), and also to reduce the migration of murine lymphatic ECs (22). In the current study, HUVEC migration was examined through Boyden transwell assays, wound healing assays and ECIS analysis. Results from all three methods confirmed the inhibitory role of DHA on EC migration, which may significantly contribute towards the antiangiogenetic effects.

Initiation of cell migration in response to cytokines, including VEGF, is a highly controlled process requiring the co-operation of signaling pathways, involving the extracellular matrix, transmembrane receptors, such as integrins, and actin cytoskeleton-associated motile apparatus $(23,24)$. MAPK pathways are important components of the signaling network, transducing the migratory signals generated by VEGF and playing an important role in the regulation of angiogenesis (12). VEGF activates the p38 MAPK pathway via KDR/Flk-1 in ECs, while SB203580 induces the inhibition of p38 activity and cell migration (12). Artemisinin and its derivatives have been shown to increase or suppress p38 MAPK activity in different experimental settings $(25,26)$. In the present study, DHA was shown to not affect the VEGF-induced activation of p38 MAPK, indicating that DHA exerts antimigratory effects independent of the p38 MAPK pathway.
The ECIS system provides a highly sensitive method to monitor real-time cell migration (27). Consistent with previous observations, ECIS analysis revealed that SB203850 significantly inhibited cell migration. However, the application of DHA to SB203850-treated HUVECs resulted in an additional decrease in cell migration. Thus, inhibiting the p38 MAPK pathway failed to prevent the DHA-induced reduction of cell migration. These results further confirm that p38 MAPK does not mediate the DHA-induced inhibition of EC migration.

Although p38 MAPK plays a key role in promoting cell migration, other signaling pathways are also involved in regulating this process. The nuclear factor- $\kappa \mathrm{B}(\mathrm{NF}-\kappa \mathrm{B})$ family comprises a wide range of transcription factors that are involved in numerous cellular functions (28). Activation of the $\mathrm{NF}-\kappa \mathrm{B}$ pathway promotes cell migration. Extracellular stimuli, such as high glucose concentrations, inhibit EC migration via the suppression of the NF- $\kappa \mathrm{B}$ pathway (29). DHA inhibits the $\mathrm{NF}-\kappa \mathrm{B}$ pathway by preventing the nuclear translocation of the p65/p50 complex (30). In addition, the protein kinase B (PKB)signaling pathway positively regulates cell migration, and oxidized low-density lipoprotein inhibits EC migration through the suppression of this pathway (31). Artemisinin and its derivatives have been shown to inhibit PKB activity (32). Therefore, the DHA-induced inhibition of EC migration may be mediated by these $\mathrm{p} 38$-independent signaling pathways.

In conclusion, the results of the present study demonstrated that DHA inhibits EC migration, which is independent of p38 
MAPK signaling. These observations provide an insight into the mechanisms underlying the antiangiogenetic effects of the artemisinin family of drugs, which may develop into therapeutic agents against cancer and other vascular-associated diseases.

\section{Acknowledgements}

This study was supported by a grant from the Medical Science and Technology Development Plan of Shandong Province, China (no. 2013WS0137). The authors are grateful for the financial support from the Shandong Taishan Scholarship (Ju Liu).

\section{References}

1. Klayman DL: Qinghaosu (artemisinin): an antimalarial drug from China. Science 228: 1049-1055, 1985

2. Tu Y: The development of new antimalarial drugs: qinghaosu and dihydro-qinghaosu. Chin Med J (Engl) 112: 976-977, 1999.

3. Gordi T and Lepist EI: Artemisinin derivatives: toxic for laboratory animals, safe for humans? Toxicol Lett 147: 99-107, 2004.

4. Crespo-Ortiz MP and Wei MQ: Antitumor activity of artemisinin and its derivatives: from a well-known antimalarial agent to a potential anticancer drug. J Biomed Biotechnol 2012: 247597, 2012.

5. Risau W: Mechanisms of angiogenesis. Nature 386: 671-674, 1997.

6. Costa C, Soares R and Schmitt F: Angiogenesis: now and then. APMIS 112: 402-412, 2004.

7. Klagsbrun M and Moses MA: Molecular angiogenesis. Chem Biol 6: R217-R224, 1999.

8. Steeg PS: Tumor metastasis: mechanistic insights and clinical challenges. Nat Med 12: 895-904, 2006.

9. Folkman J: Angiogenesis in cancer, vascular, rheumatoid and other disease. Nat Med 1: 27-31, 1995.

10. Hoefen RJ and Berk BC: The role of MAP kinases in endothelial activation. Vascul Pharmacol 38: 271-273, 2002.

11. Page C and Doubell AF: Mitogen-activated protein kinase (MAPK) in cardiac tissues. Mol Cell Biochem 157: 49-57, 1996.

12. Rousseau S, Houle F, Landry J and Huot J: p38 MAP kinase activation by vascular endothelial growth factor mediates actin reorganization and cell migration in human endothelial cells. Oncogene 15: 2169-2177, 1997.

13. Harris VK, Coticchia CM, Kagan BL, Ahmad S, Wellstein A and Riegel AT: Induction of the angiogenic modulator fibroblast growth factor-binding protein by epidermal growth factor is mediated through both MEK/ERK and p38 signal transduction pathways. J Biol Chem 275: 10802-10811, 2000.

14. Wang SJ, Sun B, Cheng ZX, et al: Dihydroartemisinin inhibits angiogenesis in pancreatic cancer by targeting the NF- $\kappa \mathrm{B}$ pathway. Cancer Chemother Pharmacol 68: 1421-1430, 2011.

15. Liang CC, Park AY and Guan JL: In vitro scratch assay: a convenient and inexpensive method for analysis of cell migration in vitro. Nat Protoc 2: 329-333, 2007.
16. Liu J and Kapron CM: Differential induction of MAP kinase signalling pathways by cadmium in primary cultures of mouse embryo limb bud cells. Reprod Toxicol 29: 286-291, 2010.

17. Cuenda A, Rouse J, Doza YN, et al: SB 203580 is a specific inhibitor of a MAP kinase homologue which is stimulated by cellular stresses and interleukin-1. FEBS Lett 364: 229-233, 1995.

18. Vasudev NS and Reynolds AR: Anti-angiogenic therapy for cancer: current progress, unresolved questions and future directions. Angiogenesis 17: 471-494, 2014.

19. Lamalice L, Le Boeuf F and Huot J: Endothelial cell migration during angiogenesis. Circ Res 100: 782-794, 2007.

20. Zhang JL, Wang Z, Hu W, Chen SS, Lou XE and Zhou HJ: DHA regulates angiogenesis and improves the efficiency of CDDP for the treatment of lung carcinoma. Microvasc Res 87: 14-24, 2013.

21. Cheng R, Li C, Li C, et al: The artemisinin derivative artesunate inhibits corneal neovascularization by inducing ROS-dependent apoptosis in vascular endothelial cells. Invest Ophthalmol Vis Sci 54: 3400-3409, 2013.

22. Wang J, Guo Y, Zhang BC, Chen ZT and Gao JF: Induction of apoptosis and inhibition of cell migration and tube-like formation by dihydroartemisinin in murine lymphatic endothelial cells. Pharmacology 80: 207-218, 2007.

23. Nobes CD and Hall A: Rho, rac, and cdc42 GTPases regulate the assembly of multimolecular focal complexes associated with actin stress fibers, lamellipodia, and filopodia. Cell 81: 53-62, 1995.

24. Lauffenburger DA and Horwitz AF: Cell migration: a physically integrated molecular process. Cell 84: 359-369, 1996.

25. Wang JX, Hou LF, Yang Y, Tang W, Li Y and Zuo JP: SM905, an artemisinin derivative, inhibited $\mathrm{NO}$ and pro-inflammatory cytokine production by suppressing MAPK and NF-kappaB pathways in RAW 264.7 macrophages. Acta Pharmacol Sin 30: $1428-1435,2009$

26. Lu JJ, Meng LH, Cai YJ, et al: Dihydroartemisinin induces apoptosis in HL-60 leukemia cells dependent of iron and p38 mitogen-activated protein kinase activation but independent of reactive oxygen species. Cancer Biol Ther 7: 1017-1023, 2008.

27. Hong J, Kandasamy K, Marimuthu M, Choi CS and Kim S: Electrical cell-substrate impedance sensing as a non-invasive tool for cancer cell study. Analyst 136: 237-245, 2011.

28. Jacobs MD and Harrison SC: Structure of an IkappaBalpha/NF-kappaB complex. Cell 95: 749-758, 1998.

29. Hamuro M, Polan J, Natarajan M and Mohan S: High glucose induced nuclear factor kappa B mediated inhibition of endothelial cell migration. Atherosclerosis 162: 277-287, 2002.

30. Hwang YP, Yun HJ, Kim HG, Han EH, Lee GW and Jeong HG: Suppression of PMA-induced tumor cell invasion by dihydroartemisinin via inhibition of PKCalpha/Raf/MAPKs and NF-kappaB/AP-1-dependent mechanisms. Biochem Pharmacol 79: 1714-1726, 2010.

31. Chavakis E, Dernbach E, Hermann C, Mondorf UF, Zeiher AM and Dimmeler S: Oxidized LDL inhibits vascular endothelial growth factor-induced endothelial cell migration by an inhibitory effect on the Akt/endothelial nitric oxide synthase pathway. Circulation 103: 2102-2107, 2001.

32. Cheng C, Ho WE, Goh FY, et al: Anti-malarial drug artesunate attenuates experimental allergic asthma via inhibition of the phosphoinositide 3-kinase/Akt pathway. PloS One 6: e20932, 2011. 